\title{
Domination Integrity of Splitting Graph of Path and Cycle
}

\author{
Samir K. Vaidya ${ }^{1}$ and Nirang J. Kothari ${ }^{2}$ \\ ${ }^{1}$ Department of Mathematics, Saurashtra University, Rajkot 360005, Gujarat, India \\ ${ }^{2}$ B. H. Gardi College of Engineering and Technology, P.O. Box 215, Rajkot 360001, Gujarat, India
}

Correspondence should be addressed to Samir K. Vaidya; samirkvaidya@yahoo.co.in

Received 9 October 2012; Accepted 19 November 2012

Academic Editors: C. da Fonseca, R. Dondi, and R. Sotirov

Copyright (C) 2013 S. K. Vaidya and N. J. Kothari. This is an open access article distributed under the Creative Commons Attribution License, which permits unrestricted use, distribution, and reproduction in any medium, provided the original work is properly cited.

If $S$ is a dominating set of a connected graph $G$ then the domination integrity is the minimum of the sum of two parameters, the number of elements in $S$ and the order of the maximum component of $G-S$. We investigate domination integrity of splitting graph of path $P_{n}$ and cycle $C_{n}$. This work is an effort to relate network expansion and vulnerability parameter.

\section{Introduction}

The stability of a communication network is of prime importance for any network designer. A shrinking network eventually loses links or nodes, its effectiveness is continuously decreasing and network becomes vulnerable. Many graph theoretic parameters have been introduced for the measurement of vulnerability. Some of them are connectivity, toughness, integrity, and rupture degree. The integrity of a graph is one of the well explored concepts which was introduced by Barefoot et al. [1].

Definition 1. The integrity of a graph $G$ is denoted by $I(G)$ and defined by $I(G)=\min \{|S|+m(G-S): S \subset V(G)\}$, where $m(G-S)$ is the order of a maximum component of $G-S$.

Definition 2. An $I$-set of $G$ is any (proper) subset $S$ of $V(G)$ for which $I(G)=|S|+m(G-S)$.

The integrity of the complete graph $K_{n}$, path $P_{n}$, cycle $C_{n}$, star $K_{1, n}$, complete bipartite graph $K_{m, n}$, and power graph of cycle were discussed by Barefoot et al. $[1,2]$ while Goddard and Swart [3,4] have investigated the bounds for integrity of graphs and its complement. They have also investigated the integrity of graphs in the context of some graph operations. The integrity of middle graphs is discussed by Mamut and
Vumar [5] while integrity of total graphs is discussed by Dündar and Aytaç [6].

Definition 3. A set $S \subseteq V$ of vertices in a graph $G=(V, E)$ is called dominating set if every vertex $v \in V$ is either an element of $S$ or is adjacent to an element of $S$.

Definition 4. The domination number $\gamma(G)$ of a graph $G$ equals the minimum cardinality of minimal dominating set of graph $G$.

If $D$ is any minimal dominating set and if the order of the largest component of $G-D$ is small, then the removal of $D$ will crash the communication network. Considering this aspect, the concept of domination integrity was introduced by Sundareswaran and Swaminathan [7].

Definition 5. The domination integrity of a connected graph $G$ is denoted as $D I(G)=\min \{|S|+m(G-S): S$ is a dominating set\}, where $m(G-S)$ is the order of a maximum component of $G-S$.

Sundareswaran and Swaminathan [8] have investigated domination integrity of middle graph of some graphs while Vaidya and Kothari [9] have discussed domination integrity of a graph obtained by duplication of an edge by a vertex and 
duplication of vertex by an edge in $C_{n}$ and $P_{n}$. In the present work we investigate domination integrity of splitting graphs of path and cycle.

Definition 6. For a graph $G$ the splitting graph $S^{\prime}(G)$ of graph $G$ is obtained by adding a new vertex $v$ ' corresponding to each vertex $v$ of $G$ such that $N(v)=N\left(v^{\prime}\right)$ where $N(v)$ and $N\left(v^{\prime}\right)$ are the neighborhood sets of $v$ and $v$, respectively.

For standard graph theoretic notation we refer to West [10] while for terminology related to domination in Graphs we refer to Haynes et al. [11].

\section{Main Results}

Theorem 7. For all $n \geq 2$,

$$
\gamma\left(S^{\prime}\left(P_{n}\right)\right)= \begin{cases}\frac{n}{2} & \text { if } n \equiv 0(\bmod 4) \\ \frac{n+1}{2} & \text { if } n \equiv 1,3(\bmod 4) \\ \frac{n+2}{2} & \text { if } n \equiv 2(\bmod 4) .\end{cases}
$$

Proof. Let $v_{1}, v_{2}, \ldots, v_{n}$ be the vertices of path $P_{n}$ and $u_{1}, u_{2}, \ldots, u_{n}$ be the vertices corresponding to $v_{1}, v_{2}, \ldots, v_{n}$ which are added to obtain $S^{\prime}\left(P_{n}\right)$. As $N\left(u_{1}\right)=\left\{v_{2}\right\}$ and $N\left(u_{n}\right)=\left\{v_{n-1}\right\}$, at least one vertex from each pair from $u_{1}, v_{2}$ and $u_{n}, v_{n-1}$ must belong to any dominating set of $S^{\prime}\left(P_{n}\right)$. Also at least one vertex from $v_{i-1}$ and $v_{i+1}$ must belong to any dominating set as $N\left(u_{i}\right)=\left\{v_{i-1}, v_{i+1}\right\}$ and $N\left(v_{i}\right)=$ $\left\{v_{i-1}, v_{i+1}, u_{i-1}, u_{i+1}\right\}$, where $2 \leq i \leq n-1$. Consequently

$$
|S| \geq \frac{n}{2} \quad \text { for any dominating set } S \text {. }
$$

Now depending upon the number of vertices in $P_{n}$ we consider following subsets for $0 \leq j<\lfloor n / 4\rfloor$ :

$$
\begin{array}{r}
S=\left\{v_{2+4 j}, v_{3+4 j}\right\}, \quad|S|=\frac{n}{2} \\
\text { for } n \equiv 0(\bmod 4), \\
S=\left\{v_{2+4 j}, v_{3+4 j}, v_{n-1}\right\}, \quad|S|=\frac{n+1}{2} \\
\text { for } n \equiv 1(\bmod 4), \\
S=\left\{v_{2+4 j}, v_{3+4 j}, v_{n-2}, v_{n-1}\right\}, \quad|S|=\frac{n+2}{2} \\
\text { for } n \equiv 2(\bmod 4), \\
S=\left\{v_{2+4 j}, v_{3+4 j}, v_{n-2}, v_{n-1}\right\}, \quad|S|=\frac{n+1}{2} \\
\text { for } n \equiv 3(\bmod 4) .
\end{array}
$$

We claim that each $S$ is dominating set because $N\left(v_{2+4 j}\right)=\left\{v_{1+4 j}, v_{3+4 j}, u_{1+4 j}, u_{3+4 j}\right\}$ and $N\left(v_{3+4 j}\right)=$ $\left\{v_{2+4 j}, v_{4+4 j}, u_{2+4 j}, u_{4+4 j}\right\}$.
Also each $S$ is minimal because the vertex $u_{1+4 j}$ will not be dominated by any of the vertices when the vertex $v_{2+4 j}$ is removed.

Thus above defined $S$ is minimal dominating set of $S^{\prime}\left(P_{n}\right)$, hence from (2) we get

$$
\gamma\left(S^{\prime}\left(P_{n}\right)\right)= \begin{cases}\frac{n}{2} & \text { if } n \equiv 0(\bmod 4) \\ \frac{n+1}{2} & \text { if } n \equiv 1,3(\bmod 4) \\ \frac{n+2}{2} & \text { if } n \equiv 2(\bmod 4) .\end{cases}
$$

Observation 1. If $S$ is any dominating set of $S^{\prime}\left(P_{n}\right)$ with $m\left(S^{\prime}\left(P_{n}\right)-S\right)=2$ then $|S| \geq n-2$.

Observation 2. If $S$ is any dominating set of $S^{\prime}\left(P_{n}\right)$ with $m\left(S^{\prime}\left(P_{n}\right)-S\right)=1$ then $|S| \geq n$.

\section{Theorem 8.}

$$
D I\left(S^{\prime}\left(P_{n}\right)\right)= \begin{cases}3 & \text { if } n=2 \\ n & \text { if } 3 \leqslant n \leqslant 9 \\ n-1 & \text { if } 10 \leqslant n \leqslant 12 \\ n-2 & \text { if } 13 \leqslant n \leqslant 15 \\ n-3 & \text { if } 16 \leqslant n \leqslant 18 \\ 15 & \text { if } n=19 .\end{cases}
$$

Proof. Let $v_{1}, v_{2}, \ldots, v_{n}$ be the vertices of path $P_{n}$ and $u_{1}, u_{2}, \ldots, u_{n}$ be the vertices corresponding to $v_{1}, v_{2}, \ldots, v_{n}$ which are added to obtain $S^{\prime}\left(P_{n}\right)$.

Case $1(n=2)$. From Theorem $7 \gamma\left(S^{\prime}\left(P_{2}\right)\right)=2$ and $D=$ $\left\{v_{1}, v_{2}\right\}$ is a $\gamma$-set of $S^{\prime}\left(P_{2}\right)$. Then $m\left(S^{\prime}\left(P_{2}\right)-D\right)=1$. This implies that $D I\left(S^{\prime}\left(P_{2}\right)\right)=\gamma\left(S^{\prime}\left(P_{2}\right)\right)+m\left(S^{\prime}\left(P_{2}\right)-D\right)=2+1=$ 3 since $\gamma\left(S^{\prime}\left(P_{2}\right)\right) \leq|S|$ and $m\left(S^{\prime}\left(P_{2}\right)-D\right) \leq m\left(S^{\prime}\left(P_{2}\right)-S\right)$ for any dominating set $S$ of $S^{\prime}\left(P_{2}\right)$. Consequently $\gamma\left(S^{\prime}\left(P_{2}\right)\right)+$ $m\left(S^{\prime}\left(P_{2}\right)-D\right) \leq|S|+m\left(S^{\prime}\left(P_{2}\right)-S\right)$ for any dominating set $S$ of $S^{\prime}\left(P_{2}\right)$. Hence $D I\left(S^{\prime}\left(P_{2}\right)\right)=3$.

Case $2(3 \leq n \leq 9)$. Consider the following subcases.

Subcase (i) $(n=3)$. From Theorem $7 \gamma\left(S^{\prime}\left(P_{3}\right)\right)=2$ and $D=$ $\left\{v_{2}, u_{2}\right\}$ is a $\gamma$-set of $S^{\prime}\left(P_{3}\right)$. Then $m\left(S^{\prime}\left(P_{3}\right)-D\right)=1$. This implies that $D I\left(S^{\prime}\left(P_{3}\right)\right)=\gamma\left(S^{\prime}\left(P_{3}\right)\right)+m\left(S^{\prime}\left(P_{3}\right)-D\right)=2+1=$ 3 since $\gamma\left(S^{\prime}\left(P_{3}\right)\right) \leq|S|$ and $m\left(S^{\prime}\left(P_{3}\right)-D\right) \leq m\left(S^{\prime}\left(P_{3}\right)-S\right)$ for any dominating set $S$ of $S^{\prime}\left(P_{3}\right)$. Consequently $\gamma\left(S^{\prime}\left(P_{3}\right)\right)+$ $m\left(S^{\prime}\left(P_{3}\right)-D\right) \leq|S|+m\left(S^{\prime}\left(P_{3}\right)-S\right)$ for any dominating set $S$ of $S^{\prime}\left(P_{3}\right)$. Hence $D I\left(S^{\prime}\left(P_{3}\right)\right)=3$.

Subcase (ii) $(4 \leq n \leq 6)$. From Theorem $7 \gamma\left(S^{\prime}\left(P_{n}\right)\right)=n-2$ and $D=\left\{v_{2}, v_{3}, \ldots, v_{n-1}\right\}$ is a $\gamma$-set of $S^{\prime}\left(P_{n}\right)$. Then $m\left(S^{\prime}\left(P_{n}\right)-\right.$ D) $=2$. 
Therefore

$$
\begin{aligned}
D I\left(S^{\prime}\left(P_{n}\right)\right) & \leq \gamma\left(S^{\prime}\left(P_{n}\right)\right)+m\left(S^{\prime}\left(P_{n}\right)-D\right) \\
& =n-2+2=n .
\end{aligned}
$$

If $S$ is any dominating set other than $D$ of $S^{\prime}\left(P_{n}\right)$ with $m\left(S^{\prime}\left(P_{n}\right)-S\right)=1$ then $|S| \geq n$. This implies that

$$
|S|+m\left(S^{\prime}\left(P_{n}\right)-S\right) \geq n+1>n .
$$

Hence from (6) and (7) $D I\left(S^{\prime}\left(P_{n}\right)\right)=n$.

Subcase (iii) $(n=7)$. From Theorem $7 \gamma\left(S^{\prime}\left(P_{7}\right)\right)=4$ and $D=$ $\left\{v_{2}, v_{3}, v_{5}, v_{6}\right\}$ is a $\gamma$-set of $S^{\prime}\left(P_{7}\right)$. Then $m\left(S^{\prime}\left(P_{7}\right)-D\right)=3$.

\section{Therefore}

$$
D I\left(S^{\prime}\left(P_{7}\right)\right) \leq \gamma\left(S^{\prime}\left(P_{7}\right)\right)+m\left(S^{\prime}\left(P_{7}\right)-D\right)=4+3=7 .
$$

Now if $S_{1}$ is any dominating set other than $D$ of $S^{\prime}\left(P_{7}\right)$ and $m\left(S^{\prime}\left(P_{7}\right)-S_{1}\right)=2$ then $\left|S_{1}\right| \geq 5$. This implies that

$$
\left|S_{1}\right|+m\left(S^{\prime}\left(P_{7}\right)-S_{1}\right) \geq 5+2=7 \text {. }
$$

Also if $S_{2}$ is any dominating set other than $D$ and $S_{1}$ of $S^{\prime}\left(P_{7}\right)$ with $m\left(S^{\prime}\left(P_{7}\right)-S_{2}\right)=1$ then $\left|S_{2}\right| \geq 7$.

This implies that

$$
\left|S_{2}\right|+m\left(S^{\prime}\left(P_{7}\right)-S_{2}\right) \geq 7+1=8
$$

Hence from (8), (9), and (10) DI $\left(S^{\prime}\left(P_{7}\right)\right)=7$.

Case $3(n=10)$. From Theorem $7 \gamma\left(S^{\prime}\left(P_{10}\right)\right)=6$ and $D=$ $\left\{v_{2}, v_{3}, v_{5}, v_{6}, v_{8}, v_{9}\right\}$ is a $\gamma$-set of $S^{\prime}\left(P_{10}\right)$. Then $m\left(S^{\prime}\left(P_{10}\right)-\right.$ D) $=3$.

Therefore

$$
\begin{aligned}
D I\left(S^{\prime}\left(P_{10}\right)\right) & \leq \gamma\left(S^{\prime}\left(P_{10}\right)\right)+m\left(S^{\prime}\left(P_{10}\right)-D\right) \\
& =6+3=9 .
\end{aligned}
$$

Now if $S_{1}$ is any dominating set other than $D$ of $S^{\prime}\left(P_{10}\right)$ and $m\left(S^{\prime}\left(P_{10}\right)-S_{1}\right)=2$ then $\left|S_{1}\right| \geq 8$.

This implies that

$$
\left|S_{1}\right|+m\left(S^{\prime}\left(P_{10}\right)-S_{1}\right) \geq 8+2=10 .
$$

Also if $S_{2}$ is any dominating set other than $D$ and $S_{1}$ of $S^{\prime}\left(P_{10}\right)$ with $m\left(S^{\prime}\left(P_{10}\right)-S_{2}\right)=1$ then $\left|S_{2}\right| \geq 10$.

This implies that

$$
\left|S_{2}\right|+m\left(S^{\prime}\left(P_{10}\right)-S_{2}\right) \geq 10+1=11 \text {. }
$$

Hence from (11), (12) and (13) $D I\left(S^{\prime}\left(P_{10}\right)\right)=9$.

Case $4(n=8,9$ and $11 \leq n \leq 19)$. We know that

$$
D I\left(S^{\prime}\left(P_{n}\right)\right) \leq \gamma\left(S^{\prime}\left(P_{n}\right)\right)+m\left(S^{\prime}\left(P_{n}\right)-D\right) .
$$

The domination number with $\gamma\left(S^{\prime}\left(C_{n}\right)\right)+m\left(S^{\prime}\left(P_{n}\right)-D\right)$ and set $S$ with $|S|+m\left(S^{\prime}\left(P_{n}\right)-S\right)$ are shown in Table 1 .

From Table 1

$$
|S|+m\left(S^{\prime}\left(P_{n}\right)-S\right) \leq \gamma\left(S^{\prime}\left(P_{n}\right)\right)+m\left(S^{\prime}\left(P_{n}\right)-D\right) \text {. }
$$

If $S_{1}$ is any dominating set of $S^{\prime}\left(P_{n}\right)$ other than $D$ and $S$ with $m\left(S^{\prime}\left(P_{n}\right)-S_{1}\right)=4$ or 5 then $|S| \leq\left|S_{1}\right|$.

This implies that

$$
|S|+m\left(S^{\prime}\left(P_{n}\right)-S\right) \leq\left|S_{1}\right|+m\left(S^{\prime}\left(P_{n}\right)-S_{1}\right) .
$$

If $S_{2}$ is any dominating set other than $D, S_{1}$, and $S$ with $m\left(S^{\prime}\left(P_{n}\right)-S_{2}\right)=2$ then $\left|S_{2}\right| \geq n-2$.

This implies that

$$
\left|S_{2}\right|+m\left(S^{\prime}\left(P_{n}\right)-S_{2}\right) \geq(n-2)+2=n .
$$

If $S_{3}$ is any dominating set other than $D, S_{1}, S_{2}$, and $S$ with $m\left(S^{\prime}\left(P_{n}\right)-S_{3}\right)=1$ then $\left|S_{3}\right| \geq n$.

This implies that

$$
\left|S_{3}\right|+m\left(S^{\prime}\left(P_{n}\right)-S_{3}\right) \geq n+1 .
$$

Hence from Table 1 and the results (14) to (18) we conclude that

$$
D I\left(S^{\prime}\left(P_{n}\right)\right)=|S|+m\left(S^{\prime}\left(P_{n}\right)-S\right)
$$

$$
= \begin{cases}3 & \text { if } n=2 \\ n & \text { if } 3 \leqslant n \leqslant 9 \\ n-1 & \text { if } 10 \leqslant n \leqslant 12 \\ n-2 & \text { if } 13 \leqslant n \leqslant 15 \\ n-3 & \text { if } 16 \leqslant n \leqslant 18 \\ 15 & \text { if } n=19 .\end{cases}
$$

Hence the result.

\section{Theorem 9}

$$
D I\left(S^{\prime}\left(P_{n}\right)\right)=\gamma\left(S^{\prime}\left(P_{n}\right)\right)+6, \quad \forall n \geq 20 .
$$

Proof. Let $v_{1}, v_{2}, \ldots, v_{n}$ be the vertices of path $P_{n}$ and $u_{1}, u_{2}, \ldots, u_{n}$ be the vertices corresponding to $v_{1}, v_{2}, \ldots, v_{n}$ which are added to obtain $S^{\prime}\left(P_{n}\right)$. Then from Theorem 7

$$
\gamma\left(S^{\prime}\left(P_{n}\right)\right)= \begin{cases}\frac{n}{2} & \text { if } n \equiv 0(\bmod 4) \\ \frac{n+1}{2} & \text { if } n \equiv 1,3(\bmod 4) \\ \frac{n+2}{2} & \text { if } n \equiv 2(\bmod 4),\end{cases}
$$


TABLE 1

\begin{tabular}{|c|c|c|c|c|c|c|}
\hline$n$ & $\gamma\left(S^{\prime}\left(P_{n}\right)\right)$ & $m\left(S^{\prime}\left(P_{n}\right)-D\right)$ & $\gamma\left(S^{\prime}\left(P_{n}\right)\right)+m\left(S^{\prime}\left(P_{n}\right)-D\right)$ & $S$ & $m\left(S^{\prime}\left(P_{n}\right)-S\right)$ & $|S|+m\left(S^{\prime}\left(P_{n}\right)-S\right)$ \\
\hline 8 & 4 & 6 & 10 & $\left\{v_{2}, v_{3}, v_{4}, v_{6}, v_{7}\right\}$ & 3 & 8 \\
\hline 9 & 5 & 6 & 11 & $\left\{v_{2}, v_{3}, v_{5}, v_{6}, v_{7}, v_{8}\right\}$ & 3 & 9 \\
\hline 11 & 6 & 6 & 12 & $\left\{v_{2}, v_{3}, v_{5}, v_{6}, v_{7}, v_{9}, v_{10}\right\}$ & 3 & 10 \\
\hline 12 & 6 & 6 & 12 & $\left\{v_{2}, v_{3}, v_{5}, v_{6}, v_{7}, v_{8}, v_{10}, v_{11}\right\}$ & 3 & 11 \\
\hline 13 & 7 & 6 & 13 & $\left\{v_{2}, v_{3}, v_{5}, v_{6}, v_{8}, v_{9}, v_{11}, v_{12}\right\}$ & 3 & 11 \\
\hline 14 & 8 & 6 & 14 & $\left\{v_{2}, v_{3}, v_{4}, v_{6}, v_{7}, v_{9}, v_{10}, v_{12}, v_{13}\right\}$ & 3 & 12 \\
\hline 15 & 8 & 6 & 14 & $\left\{v_{2}, v_{3}, v_{4}, v_{6}, v_{7}, v_{8}, v_{10}, v_{11}, v_{13}, v_{14}\right\}$ & 3 & 13 \\
\hline 16 & 8 & 6 & 14 & $\left\{v_{2}, v_{3}, v_{5}, v_{6}, v_{8}, v_{9}, v_{11}, v_{12}, v_{14}, v_{15}\right\}$ & 3 & 13 \\
\hline 17 & 9 & 6 & 15 & $\left\{v_{2}, v_{3}, v_{5}, v_{6}, v_{8}, v_{9}, v_{11}, v_{12}, v_{14}, v_{15}, v_{16}\right\}$ & 3 & 14 \\
\hline 18 & 10 & 6 & 16 & $\left\{v_{2}, v_{3}, v_{5}, v_{6}, v_{8}, v_{9}, v_{11}, v_{12}, v_{14}, v_{15}, v_{16}, v_{17}\right\}$ & 3 & 15 \\
\hline 19 & 10 & 6 & 16 & $\left\{v_{2}, v_{3}, v_{5}, v_{6}, v_{8}, v_{9}, v_{11}, v_{12}, v_{14}, v_{15}, v_{17}, v_{18}\right\}$ & 3 & 15 \\
\hline
\end{tabular}

$D=\left\{v_{4 j+2}, v_{4 j+3}\right\}$ where $0 \leq j<\lfloor n / 4\rfloor$ for $n \equiv 0(\bmod 4)$, $D=\left\{v_{4 j+2}, v_{4 j+3}, v_{n-1}\right\}$ where $0 \leq j<\lfloor n / 4\rfloor-1$ for $n \equiv$ $1(\bmod 4), D=\left\{v_{4 j+2}, v_{4 j+3}, v_{n-2}, v_{n-1}\right\}$ where $0 \leq j<\lfloor n / 4\rfloor$ for $n \equiv 2,3(\bmod 4)$ are $\gamma$-sets of $S^{\prime}\left(P_{n}\right)$. Then $m\left(S^{\prime}\left(P_{n}\right)-\right.$ D) $=6$.

Therefore

$$
D I\left(S^{\prime}\left(P_{n}\right)\right) \leq \gamma\left(S^{\prime}\left(P_{n}\right)\right)+6 .
$$

Now we claim that if $m(G-S) \neq 6$ for any dominating set $S$ other than $D$ then

$$
|S|+m\left(S^{\prime}\left(P_{n}\right)-S\right) \geq \gamma\left(S^{\prime}\left(P_{n}\right)\right)+6 .
$$

Case 1. If $S_{1}$ is any dominating set other than $D$ and $m\left(S^{\prime}\left(P_{n}\right)-S_{1}\right)>4$ or 5 then $\left|S_{1}\right| \geq \gamma\left(S^{\prime}\left(P_{n}\right)\right)+2$. Consequently $\left|S_{1}\right|+m\left(S^{\prime}\left(P_{n}\right)-S_{1}\right) \geq \gamma\left(S^{\prime}\left(P_{n}\right)\right)+6$.

Case 2. If $S_{2}$ is any dominating set other than $D$ and $S_{1}$ of $S^{\prime}\left(P_{n}\right)$ with $m\left(S^{\prime}\left(P_{n}\right)-S_{2}\right)=3$ then $\left|S_{2}\right| \geq(n-\lceil n / 3\rceil)$. Consequently $\left|S_{2}\right|+m\left(S^{\prime}\left(P_{n}\right)-S_{2}\right) \geq(n-\lceil n / 3\rceil)+3>$ $(\lceil n / 2\rceil+1)+6=\gamma\left(S^{\prime}\left(P_{n}\right)\right)+6$.

Case 3. If $S_{3}$ is any dominating set other than $D, S_{1}$ and $S_{2}$ of $S^{\prime}\left(P_{n}\right)$ with $m\left(S^{\prime}\left(P_{n}\right)-S_{3}\right)=2$ then $\left|S_{3}\right| \geq n-2$. Consequently $\left|S_{3}\right|+m\left(S^{\prime}\left(P_{n}\right)-S_{3}\right) \geq(n-2)+2=n>(\lceil n / 2\rceil+1)+6=$ $\gamma\left(S^{\prime}\left(P_{n}\right)\right)+6$.

Case 4. If $S_{4}$ is any dominating set other than $D, S_{1}, S_{2}$ and $S_{3}$ of $S^{\prime}\left(P_{n}\right)$ with $m\left(S^{\prime}\left(P_{n}\right)-S_{4}\right)=1$ then $\left|S_{4}\right| \geq n$. Consequently $\left|S_{4}\right|+m\left(S^{\prime}\left(P_{n}\right)-S_{4}\right) \geq n+1>(\lceil n / 2\rceil+1)+6=\gamma\left(S^{\prime}\left(P_{n}\right)\right)+6$. 6.

Thus from (22) and (23) we have DI $\left(S^{\prime}\left(P_{n}\right)\right)=\gamma\left(S^{\prime}\left(P_{n}\right)\right)+$

Theorem 10. For all $n \geq 3$

$$
\gamma\left(S^{\prime}\left(C_{n}\right)\right)= \begin{cases}\frac{n}{2} & \text { if } n \equiv 0(\bmod 4) \\ \frac{n+1}{2} & \text { if } n \equiv 1,3(\bmod 4) \\ \frac{n+2}{2} & \text { if } n \equiv 2(\bmod 4) .\end{cases}
$$

Proof. Let $v_{1}, v_{2}, \ldots, v_{n}$ be the vertices of cycle $C_{n}$ and $u_{1}, u_{2}, \ldots, u_{n}$ be the vertices corresponding to $v_{1}, v_{2}, \ldots, v_{n}$ which are added to obtain $S^{\prime}\left(C_{n}\right)$. Now either of the vertex from $v_{i-1}$ and $v_{i+1}$ must belong to any dominating set $S$ as $N\left(u_{i}\right)=\left\{v_{i-1}, v_{i+1}\right\}$.

Consequently

$$
|S| \geq \frac{n}{2} \text { for any dominating set } S .
$$

Now depending upon the different possibilities of $n$ we choose $S$ as follows.

For $n \equiv 0(\bmod 4), S=\left\{v_{1}, v_{4 j}, v_{4 j+1}, v_{n}\right\}$ with $|S|=$ $n / 2$ (where $1 \leq j<\lfloor n / 4\rfloor$ ),

for $n \equiv 1(\bmod 4), S=\left\{v_{1}, v_{4 j}, v_{4 j+1}\right\}$ with $|S|=(n+$ 1)/2 (where $1 \leq j \leq\lfloor n / 4\rfloor)$,

for $n \equiv 2(\bmod 4), S=\left\{v_{1}, v_{4 j}, v_{4 j+1}, v_{n}\right\}$ with $|S|=$ $(n+2) / 2($ where $1 \leq j \leq\lfloor n / 4\rfloor)$,

for $n \equiv 3(\bmod 4), S=\left\{v_{1}, v_{4 j}, v_{4 j+1}, v_{n}\right\}$ with $|S|=$ $(n+1) / 2($ where $1 \leq j \leq\lfloor n / 4\rfloor)$.

We claim that each $S$ is a minimal dominating set of $S^{\prime}\left(C_{n}\right)$ since $N\left(v_{1}\right)=\left\{v_{2}, v_{n}, u_{2}, u_{n}\right\}, N\left(v_{4 j}\right)=$ $\left\{v_{4 j-1}, v_{4 j+1}, u_{4 j-1}, u_{4 j+1}\right\}, N\left(v_{4 j+1}\right)=\left\{v_{4 j}, v_{4 j+2}, u_{4 j}, u_{4 j+2}\right\}$ and $N\left(v_{n}\right)=\left\{v_{1}, v_{n-1}, u_{1}, u_{n-1}\right\}$ and removal of $v_{4 j}$, a vertex $u_{4 j+1}$ will not be dominated by any vertex hence from (25):

$$
\gamma\left(S^{\prime}\left(C_{n}\right)\right)= \begin{cases}\frac{n}{2} & \text { if } n \equiv 0(\bmod 4) \\ \frac{n+1}{2} & \text { if } n \equiv 1,3(\bmod 4) \\ \frac{n+2}{2} & \text { if } n \equiv 2(\bmod 4) .\end{cases}
$$

\section{Theorem 11.}

$$
D I\left(S^{\prime}\left(C_{n}\right)\right)= \begin{cases}n+1 ; & 3 \leq n \leq 6 \\ n-\left\lfloor\frac{n}{3}\right\rfloor+3 ; & 7 \leq n \leq 18 .\end{cases}
$$


Proof. Let $v_{1}, v_{2}, \ldots, v_{n}$ be the vertices of cycle $C_{n}$ and $u_{1}, u_{2}, \ldots, u_{n}$ be the vertices corresponding to $v_{1}, v_{2}, \ldots, v_{n}$ which are added to obtain $S^{\prime}\left(C_{n}\right)$.

Case $1(n=3)$. From Theorem $10 \gamma\left(S^{\prime}\left(C_{3}\right)\right)=2$ and $D=$ $\left\{v_{1}, v_{2}\right\}$ is a $\gamma$-set of $S^{\prime}\left(C_{3}\right)$ then $m\left(S^{\prime}\left(C_{3}\right)-D\right)=3$. Therefore

$$
\begin{aligned}
D I\left(S^{\prime}\left(C_{3}\right)\right) & \leq \gamma\left(s^{\prime}\left(C_{3}\right)\right)+m\left(s^{\prime}\left(C_{3}\right)-D\right) \\
& =2+3=5 .
\end{aligned}
$$

If $S_{1}$ is any dominating set other than $D$ of $S^{\prime}\left(C_{3}\right)$ with $m\left(S^{\prime}\left(C_{3}\right)-S_{1}\right)=2$ then $\left|S_{1}\right| \geq 3$. This implies that

$$
\left|S_{1}\right|+m\left(S^{\prime}\left(C_{3}\right)-S_{1}\right) \geq 2+3=5 \text {. }
$$

Let $S_{2}=\left\{v_{1}, v_{2}, v_{3}\right\}$ be dominating set of $S^{\prime}\left(C_{3}\right)$ and $m\left(S^{\prime}\left(C_{3}\right)-S_{2}\right)=1$. This implies that

$$
\left|S_{2}\right|+m\left(S^{\prime}\left(C_{3}\right)-S_{2}\right)=3+1=4 .
$$

Hence from (28), (29), and (30) DI $\left(S^{\prime} C_{3}\right)=4$.

Case $2(n=4)$. From Theorem $10 \gamma\left(S^{\prime}\left(C_{4}\right)\right)=2$ and $D=$ $\left\{v_{1}, v_{2}\right\}$ is a $\gamma$-set of $S^{\prime}\left(C_{4}\right)$ then $m\left(S^{\prime}\left(C_{4}\right)-D\right)=3$.

Therefore

$$
\begin{aligned}
D I\left(S^{\prime}\left(C_{4}\right)\right) & \leq \gamma\left(S^{\prime}\left(C_{4}\right)\right)+m\left(s^{\prime}\left(C_{4}\right)-D\right) \\
& =2+3=5 .
\end{aligned}
$$

If $S_{1}$ is any dominating set other than $D$ of $S^{\prime}\left(C_{4}\right)$ and $m\left(S^{\prime}\left(C_{4}\right)-S_{1}\right)=2$ or 1 then $\left|S_{1}\right| \geq 4$. Therefore

$$
\left|S_{1}\right|+m\left(S^{\prime}\left(C_{4}\right)-S_{1}\right) \geq 4+2=6 .
$$

Hence from (31) and (32) DI $\left(S^{\prime}\left(C_{4}\right)\right)=5$.

Case $3(n=5)$. From Theorem $10 \gamma\left(S^{\prime}\left(C_{5}\right)\right)=3$ and $D=$ $\left\{v_{2}, v_{3}, v_{4}\right\}$ is a $\gamma$-set of $S^{\prime}\left(C_{5}\right)$ then $m\left(S^{\prime}\left(C_{5}\right)-D\right)=6$.

Therefore

$$
\begin{aligned}
D I\left(S^{\prime}\left(C_{5}\right)\right) & \leq \gamma\left(S^{\prime}\left(C_{5}\right)\right)+m\left(s^{\prime}\left(C_{5}\right)-D\right) \\
& =3+6=9 .
\end{aligned}
$$

If $S_{1}$ is any dominating set other than $D$ of $S^{\prime}\left(C_{5}\right)$ and $m\left(S^{\prime}\left(C_{5}\right)-S_{1}\right)=4$ or 5 then $\left|S_{1}\right| \geq \gamma\left(S^{\prime}\left(C_{5}\right)\right)=3$.

This implies

$$
\begin{aligned}
\left|S_{1}\right|+m\left(s^{\prime}\left(C_{5}\right)-S_{1}\right) & \geq \gamma\left(s^{\prime}\left(C_{5}\right)\right)+4 \\
& =3+4=7 .
\end{aligned}
$$

If $S_{2}$ is any dominating set other than $D$ and $S_{1}$ with $m\left(S^{\prime}\left(C_{5}\right)-S_{2}\right)=2$ or 3 then $\left|S_{2}\right| \geq 5$. This implies that

$$
\left|S_{2}\right|+m\left(S^{\prime}\left(C_{5}\right)-S_{2}\right) \geq 5+2=7 \text {. }
$$

Let $S_{3}=\left\{v_{1}, v_{2}, v_{3}, v_{4}, v_{5}\right\}$ be dominating set of $S^{\prime}\left(C_{5}\right)$ then $m\left(S^{\prime}\left(C_{5}\right)-S_{3}\right)=1$. This implies that

$$
\left|S_{3}\right|+m\left(S^{\prime}\left(C_{5}\right)-S_{3}\right)=5+1=6 .
$$

Hence from (33), (34), (35), and (36) DI $\left(S^{\prime}\left(C_{5}\right)\right)=6$.

Case $4(n=6)$. From Theorem $10 \gamma\left(S^{\prime}\left(C_{6}\right)\right)=4$ and $D=$ $\left\{v_{1}, v_{2}, v_{4}, v_{5}\right\}$ is a $\gamma$-set of $S^{\prime}\left(C_{6}\right)$ then $m\left(S^{\prime}\left(C_{6}\right)-D\right)=3$.

Therefore

$$
\begin{aligned}
D I\left(s^{\prime}\left(C_{6}\right)\right) & \leq \gamma\left(s^{\prime}\left(C_{6}\right)\right)+m\left(s^{\prime}\left(C_{6}\right)-D\right) \\
& =4+3=7 .
\end{aligned}
$$

If $S_{1}$ is any dominating set other than $D$ of $S^{\prime}\left(C_{6}\right)$ and $m\left(S^{\prime}\left(C_{6}\right)-S_{1}\right)=2$ then $\left|S_{1}\right| \geq 6$. This implies that

$$
\left|S_{1}\right|+m\left(S^{\prime}\left(C_{6}\right)-S_{1}\right) \geq 6+2=8 .
$$

Let $S_{2}=\left\{v_{1}, v_{2}, v_{3}, v_{4}, v_{5}, v_{6}\right\}$ be dominating set of $S^{\prime}\left(C_{6}\right)$ then $m\left(S^{\prime}\left(C_{6}\right)-S_{2}\right)=1$.

Therefore

$$
\left|S_{2}\right|+m\left(S^{\prime}\left(C_{6}\right)-S_{2}\right)=6+1=7 .
$$

Hence from (37), (38), and (39) $D I\left(S^{\prime}\left(C_{6}\right)\right)=7$.

Case $5(7 \leq n \leq 18)$. We know that

$$
D I\left(S^{\prime}\left(C_{n}\right)\right) \leq \gamma\left(S^{\prime}\left(C_{n}\right)\right)+m\left(S^{\prime}\left(C_{n}\right)-D\right) .
$$

The domination number with $\gamma\left(S^{\prime}\left(C_{n}\right)\right)+m\left(S^{\prime}\left(C_{n}\right)-D\right)$ and set $S$ with $|S|+m\left(S\left(C_{n}\right)-S\right)$ are shown in Table 2 .

From Table 2 for any dominating set $S$ other than $D$ of $S^{\prime}\left(C_{n}\right)$, we have

$$
|S|+m\left(S^{\prime}\left(C_{n}\right)-S\right) \leq \gamma\left(S^{\prime}\left(C_{n}\right)\right)+m\left(S^{\prime}\left(C_{n}\right)-D\right) .
$$

If $S_{1}$ is any dominating set of $S^{\prime}\left(C_{n}\right)$ other than $D$ and $S$ with $m\left(S^{\prime}\left(C_{n}\right)-S_{1}\right)=4$ or 5 then $|S| \leq\left|S_{1}\right|$.

This implies that

$$
|S|+m\left(S^{\prime}\left(C_{n}\right)-S\right) \leq\left|S_{1}\right|+m\left(S^{\prime}\left(C_{n}\right)-S_{1}\right) .
$$

If $S_{2}$ is any dominating set of $S^{\prime}\left(C_{n}\right)$ other than $D, S$, and $S_{1}$ with $m\left(S\left(C_{n}\right)-S_{2}\right)=2$ or 1 then $\left|S_{2}\right| \geq n$. 
TABLE 2

\begin{tabular}{ccccccc}
\hline$n$ & $\gamma\left(S^{\prime}\left(C_{n}\right)\right)$ & $m\left(S^{\prime}\left(C_{n}\right)-D\right)$ & $\gamma\left(S^{\prime}\left(C_{n}\right)\right)+m\left(S^{\prime}\left(C_{n}\right)-D\right)$ & $S$ & $m\left(S^{\prime}\left(C_{n}\right)-S\right)$ & $|S|+m\left(S^{\prime}\left(C_{n}\right)-S\right)$ \\
\hline 7 & 4 & 6 & 10 & 10 & $\left\{v_{1}, v_{2}, v_{4}, v_{5}, v_{7}\right\}$ & 3 \\
8 & 4 & 6 & 11 & $\left\{v_{1}, v_{2}, v_{3}, v_{5}, v_{6}, v_{8}\right\}$ & 3 & 9 \\
9 & 5 & 6 & 12 & $\left\{v_{1}, v_{2}, v_{4}, v_{5}, v_{7}, v_{8}\right\}$ & 3 & 3 \\
10 & 6 & 6 & 12 & $\left\{v_{1}, v_{2}, v_{4}, v_{5}, v_{7}, v_{8}, v_{10}\right\}$ & 3 & 10 \\
11 & 6 & 6 & 12 & 13 & $\left\{v_{1}, v_{2}, v_{4}, v_{5}, v_{7}, v_{8}, v_{10}, v_{11}\right\}$ & 3 \\
12 & 6 & 6 & 14 & $\left\{v_{1}, v_{2}, v_{4}, v_{5}, v_{7}, v_{8}, v_{10}, v_{12}\right\}$ & 3 & 11 \\
13 & 7 & 6 & 14 & $\left\{v_{1}, v_{2}, v_{4}, v_{5}, v_{7}, v_{8}, v_{10}, v_{11}, v_{13}, v_{14}\right\}$ & 3 & 12 \\
14 & 8 & 6 & 14 & $\left\{v_{1}, v_{2}, v_{4}, v_{5}, v_{7}, v_{8}, v_{10}, v_{11}, v_{13}, v_{14}\right\}$ & 3 & 13 \\
15 & 8 & 6 & $\left\{v_{1}, v_{2}, v_{4}, v_{5}, v_{7}, v_{8}, v_{10}, v_{11}, v_{13}, v_{14}, v_{16}\right\}$ & 3 \\
16 & 8 & 6 & & & 13 \\
\hline
\end{tabular}

This implies that

$$
\left|S_{2}\right|+m\left(S^{\prime}\left(C_{n}\right)-S_{2}\right) \geq n+1
$$

Hence from Table 2 and the results (40) to (43) we conclude that

$$
\begin{aligned}
D I\left(S^{\prime}\left(C_{n}\right)\right) & =|S|+m\left(S^{\prime}\left(C_{n}\right)-S\right) \\
& =n-\left\lfloor\frac{n}{3}\right\rfloor+3 .
\end{aligned}
$$

Hence the result.

Theorem 12. For all $n \geq 17 \mathrm{DI}\left(S^{\prime}\left(C_{n}\right)\right)=\gamma\left(S^{\prime} C_{n}\right)+6$.

Proof. Let $v_{1}, v_{2}, \ldots, v_{n}$ be the vertices of cycle $C_{n}$ and $u_{1}, u_{2}, \ldots, u_{n}$ be the vertices corresponding to $v_{1}, v_{2}, \ldots, v_{n}$ which are added to obtain $S^{\prime}\left(C_{n}\right)$. Then from Theorem 10

$$
\gamma\left(S^{\prime}\left(C_{n}\right)\right)= \begin{cases}\frac{n}{2} & \text { if } n \equiv 0(\bmod 4) \\ \frac{n+1}{2} & \text { if } n \equiv 1,3(\bmod 4) \\ \frac{n+2}{2} & \text { if } n \equiv 2(\bmod 4),\end{cases}
$$

$D=\left\{v_{1}, v_{4 j}, v_{4 j+1}, v_{n}\right\}$ (where $1 \leq j<\lfloor n / 4\rfloor$ ) for $n \equiv$ $0(\bmod 4), D=\left\{v_{1}, v_{4 j}, v_{4 j+1}\right\}$ (where $\left.1 \leq j \leq\lfloor n / 4\rfloor\right)$ for $n \equiv 1(\bmod 4), D=\left\{v_{1}, v_{4 j}, v_{4 j+1}, v_{n}\right\}$ (where $\left.1 \leq j \leq\lfloor n / 4\rfloor\right)$ for $n \equiv 2(\bmod 4)$ or $n \equiv 3(\bmod 4)$ are $\gamma$-sets of $S^{\prime}\left(C_{n}\right)$. Then $m\left(S^{\prime}\left(C_{n}\right)-D\right)=6$.

Therefore

$$
D I\left(S^{\prime}\left(C_{n}\right)\right) \leq \gamma\left(S^{\prime}\left(C_{n}\right)\right)+6
$$

Now we claim that if $m\left(S^{\prime}\left(C_{n}\right)\right) \neq 6$ for any dominating set $S$ other than $D$ then

$$
|S|+m\left(S^{\prime}\left(C_{n}\right)-S\right) \geq \gamma\left(S^{\prime}\left(C_{n}\right)\right)+6 .
$$

Case 1. If $S_{1}$ is any dominating set other than $D$ and $m\left(S^{\prime}\left(C_{n}\right)-S_{1}\right)=4$ or 5 then $\left|S_{1}\right| \geq \gamma\left(S^{\prime}\left(C_{n}\right)\right)+2$. Consequently $\left|S_{1}\right|+m\left(S^{\prime}\left(C_{n}\right)-S_{1}\right) \geq \gamma\left(S^{\prime}\left(C_{n}\right)\right)+6$.

Case 2. If $S_{2}$ is any dominating set other than $D$ and $S_{1}$ of $S^{\prime}\left(C_{n}\right)$ with $m\left(S^{\prime}\left(C_{n}\right)-S_{2}\right)=3$ then $\left|S_{2}\right| \geq(n-\lfloor n / 3\rfloor)$. Consequently $\left|S_{2}\right|+m\left(S^{\prime}\left(C_{n}\right)-S_{2}\right) \geq(n-\lfloor n / 3\rfloor)+3>$ $\gamma\left(S^{\prime}\left(C_{n}\right)\right)+6$.

Case 3. If $S_{3}$ is any dominating set other than $D, S_{1}$, and $S_{2}$ of $S^{\prime}\left(C_{n}\right)$ with $m\left(S^{\prime}\left(C_{n}\right)-S_{3}\right)=2$ or 1 then $\left|S_{3}\right| \geq n$. Consequently $\left|S_{3}\right|+m\left(S^{\prime}\left(C_{n}\right)-S_{3}\right) \geq n+1>\gamma\left(S^{\prime}\left(C_{n}\right)\right)+6$.

Thus from (46) and (47) we have $\operatorname{DI}\left(S^{\prime}\left(C_{n}\right)\right)$.

\section{Concluding Remarks}

The domination and vulnerability of network are two important concepts for the network security. We have studied an important measure of vulnerability known as domination integrity and investigate domination integrity of splitting graphs of path and cycle. The results reported here throw some light in the direction to find the domination integrity of larger graph obtained from the given graph.

\section{References}

[1] C. A. Barefoot, R. Entringer, and H. C. Swart, "Vulnerability in Graphs-a Comparative Survey," Journal of Combinatorial Mathematics and Combinatorial Computing, vol. 1, pp. 13-22, 1987.

[2] C. A. Barefoot, R. Entringer, and H. C. Swart, "Integrity of trees and powers of cycles," Congressus Numerantium, vol. 58, pp. 103-114, 1987.

[3] W. Goddard and H. C. Swart, "On the integrity of combinations of graphs," Journal of Combinatorial Mathematics and Combinatorial Computing, vol. 4, pp. 3-18, 1988.

[4] W. Goddard and H. C. Swart, "Integrity in graphs: bounds and basics," Journal of Combinatorial Mathematics and Combinatorial Computing, vol. 7, pp. 139-151, 1990.

[5] A. Mamut and E. Vumar, A Note on the Integrity of Middle Graphs, Lecture Notes in Computer Science, vol. 4381, Springer, New York, NY, USA, 2007. 
[6] P. Dündar and A. Aytaç, "Integrity of total graphs via certain parameters," Mathematical Notes, vol. 76, no. 5-6, pp. 665-672, 2004.

[7] R. Sundareswaran and V. Swaminathan, "Domination Integrity in graphs," in Proceedings of International conference on Mathematical and Experimental Physics, pp. 46-57, Narosa Publishing House, 2010.

[8] R. Sundareswaran and V. Swaminathan, "Domination integrity of middle graphs," in Algebra, Graph Theory and Their Applications, T. T. Chelvam, S. Somasundaram, and R. Kala, Eds., pp. 88-92, Narosa Publishing House, Delhi, India, 2010.

[9] S. Vaidya and N. Kothari, "Some new results on domination integrity of graphs," Open Journal of Discrete Mathematics, vol. 2, pp. 96-98, 2012.

[10] D. B. West, Introduction To Graph Theory, 2/e, Prentice-Hall, New Delhi, India, 2003.

[11] T. Haynes, S. Hedetniemi, and P. Slater, Fundamentals of Domination in Graphs, Marcel Dekker, New York, NY, USA, 1998. 


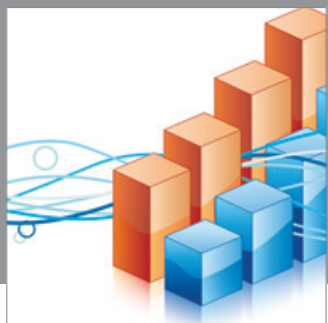

Advances in

Operations Research

mansans

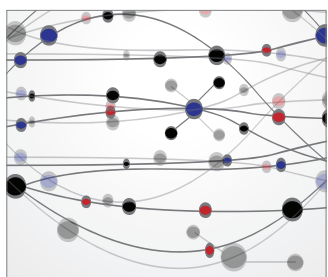

The Scientific World Journal
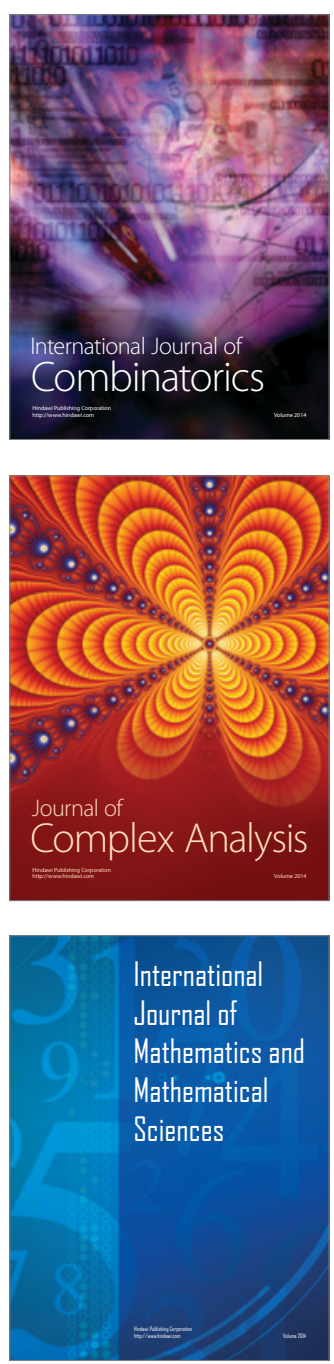
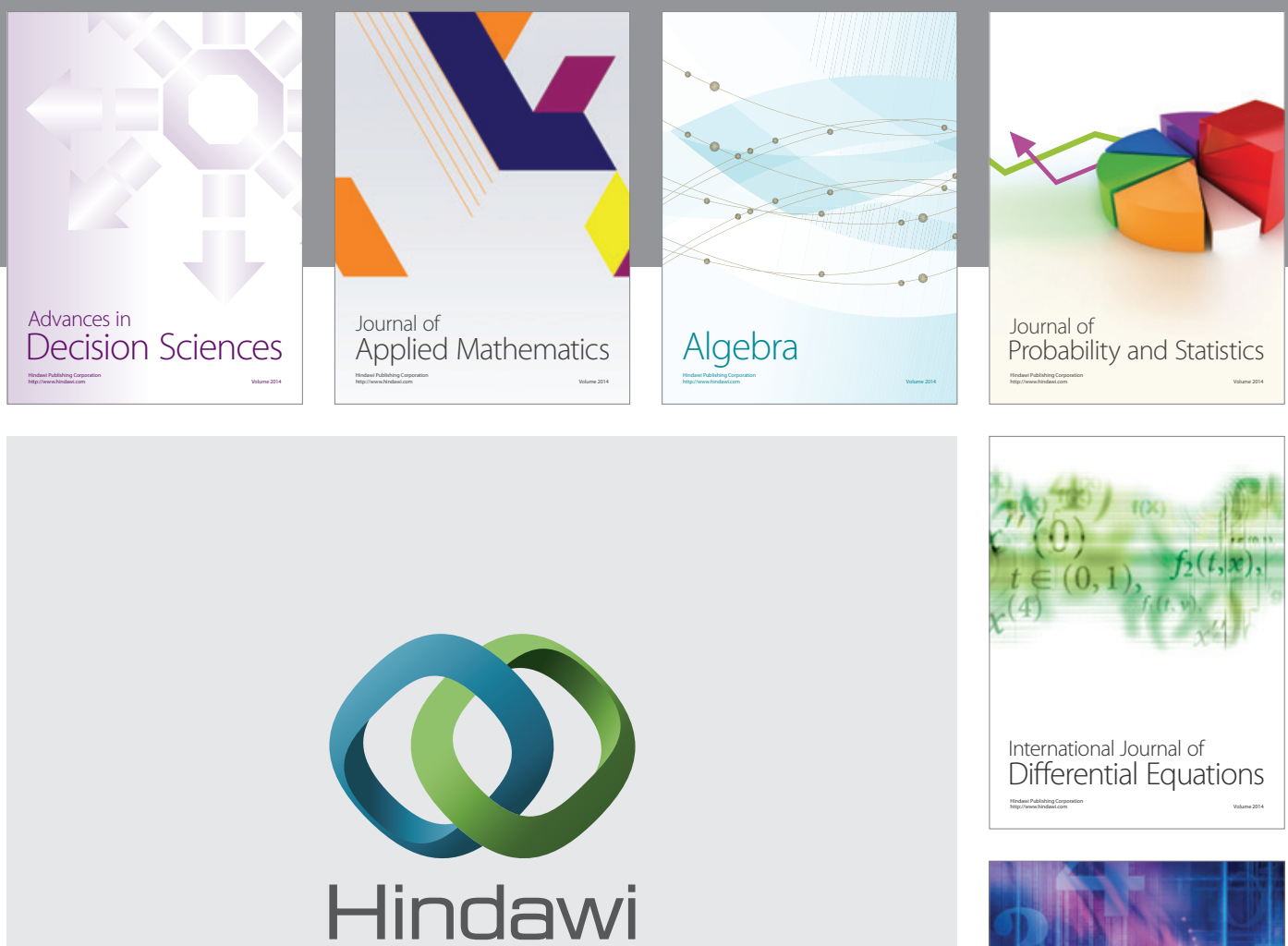

Submit your manuscripts at http://www.hindawi.com
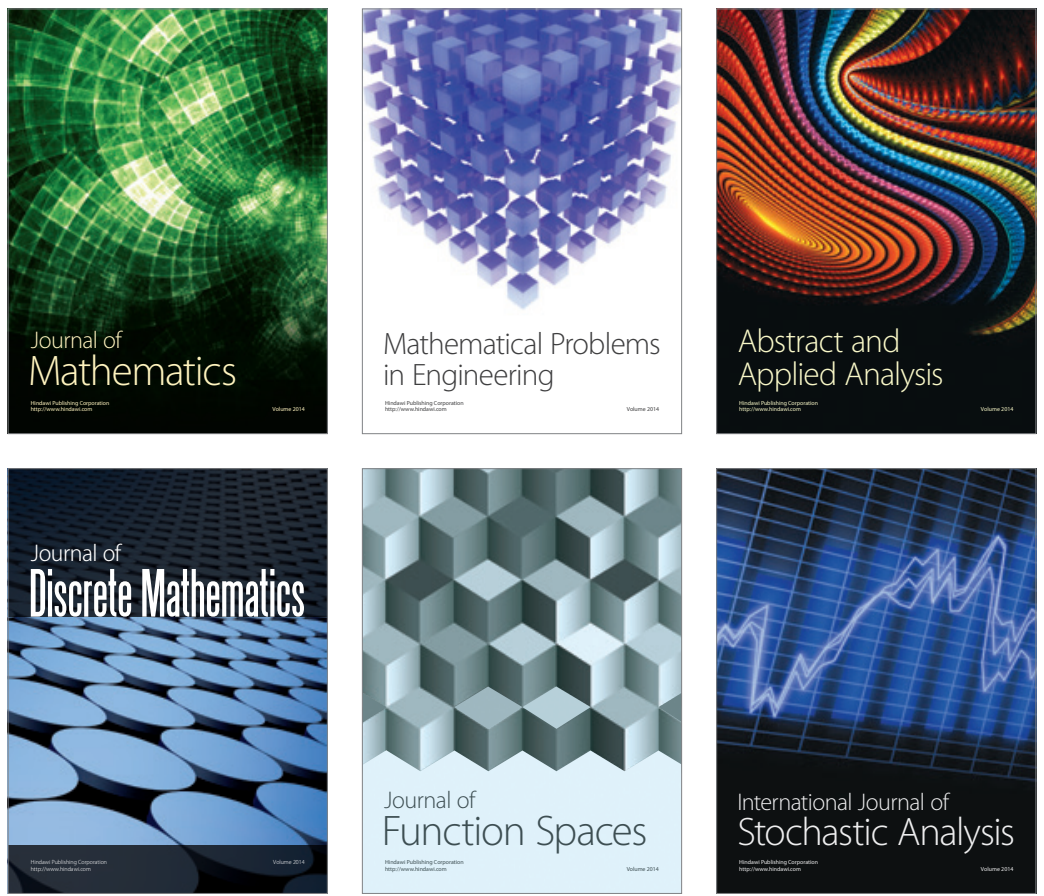

Journal of

Function Spaces

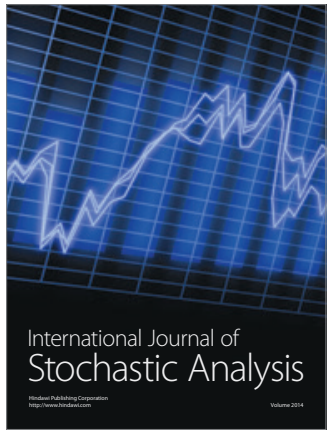

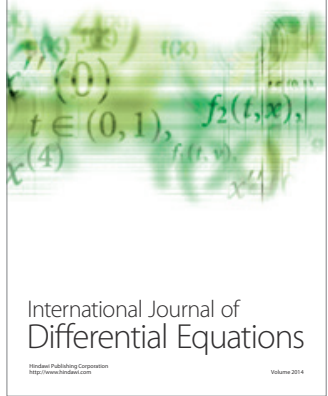
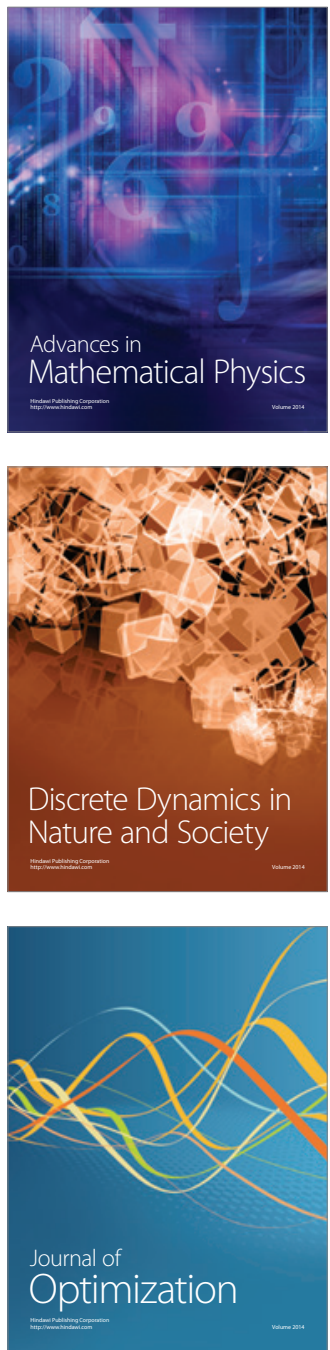